\title{
Hedyotis diffusa Willd extract suppresses Sonic hedgehog signaling leading to the inhibition of colorectal cancer angiogenesis
}

\author{
JIUMAO LIN $^{1,2}$, LIHUI WEI ${ }^{1,2}$, ALING SHEN $^{1}$, QIAOYAN CAI ${ }^{1,2}$, WEI XU $^{3}$, \\ HUANG $\mathrm{LI}^{3}$, YOUZHI ZHAN ${ }^{1,2}$, ZHENFENG HONG ${ }^{1}$ and JUN PENG ${ }^{1,2}$ \\ ${ }^{1}$ Academy of Integrative Medicine Biomedical Research Center, ${ }^{2}$ Fujian Key Laboratory \\ of Integrative Medicine on Geriatrics, ${ }^{3}$ Department of Pharmacology, Fujian University \\ of Traditional Chinese Medicine, Minhou Shangjie, Fuzhou, Fujian 350122, P.R. China
}

Received November 3, 2012; Accepted December 7, 2012

DOI: $10.3892 /$ ijo.2012.1753

\begin{abstract}
Sonic hedgehog (SHH) signaling pathway promotes the process of angiogenesis, contributing to the growth and progression of many human malignancies including colorectal cancer (CRC), which therefore has become a promising target for cancer chemotherapy. Hedyotis diffusa Willd (HDW), as a well-known traditional Chinese herbal medicine, has long been used in China for the clinic treatment of various cancers. Recently, we reported that HDW can inhibit colorectal cancer growth in vivo and in vitro via suppression of the STAT3 pathway. In addition, we demonstrated the anti-angiogenic activity of HDW in vitro. To further elucidate the mechanism of the tumoricidal activity of HDW, by using a CRC mouse xenograft model we evaluated the in vivo effect of the ethanol extract of HDW (EEHDW) on tumor angiogenesis, and investigated the underlying molecular mechanisms. We found that EEHDW could significantly reduce intratumoral microvessel density (MVD), indicating its activity of antitumor angiogenesis in vivo. EEHDW suppressed the activation of SHH signaling in CRC xenograft tumors since it significantly decreased the expression of key mediators of SHH pathway. EEHDW treatment inhibited the expression of the critical SHH signaling target gene VEGF-A as well as its specific receptor VEGFR2. Taken together, we propose for the first time that Hedyotis diffusa Willd inhibits colorectal cancer growth in vivo via inhibition of SHH-mediated tumor angiogenesis.
\end{abstract}

Correspondence to: Dr Jun Peng, Academy of Integrative Medicine Biomedical Research Center, Fujian University of Traditional Chinese Medicine, 1 Huatuo Road, Minhou Shangjie, Fuzhou, Fujian 350122, P.R. China

E-mail: pjunlab@hotmail.com

Abbreviations: EEHDW, ethanol extract of Hedyotis diffusa Willd; CRC, colorectal cancer; IHS, immunohistochemical staining; VEGF-A, vascular endothelial growth factor-A; MVD, microvessel density; SHH, sonic hedgehog signal pathway

Key words: Hedyotis diffusa Willd, Chinese medicine, colorectal cancer, angiogenesis, sonic hedgehog signal pathway

\section{Introduction}

Colorectal cancer (CRC) is one of the most common forms of cancer and a leading cause of cancer-related deaths around the world (1). To date, chemotherapy is one of the main therapeutic approaches for patients with advanced CRC $(2,3)$. However, both drug resistance and toxicity limit the effectiveness of current CRC chemotherapy, thus there is a need for the development of new therapeutic agents. Recently, natural products received great interest since they have relatively fewer side-effects and have been used clinically to treat various kinds of diseases including cancer for thousands of years. Numerous plants and their constituents have been shown to possess beneficial therapeutic effects for cancer $(4,5)$. Therefore, discovering naturally occurring agents is a promising approach for cancer chemotherapy.

Angiogenesis plays a critical role in the growth and development of cancer, suggesting that anti-angiogenic therapy is effective in inhibiting tumor progression (6-8). Tumor angiogenesis is regulated by multiple cellular signaling pathways including hedgehog $(\mathrm{HH})$ transduction cascade. $\mathrm{HH}$ pathway is important for embryonic development (9) and its aberrant activation is implicated as an initiating or maintaining factor in the progression of various human cancers (9-17). Mammals have three hedgehog homologues (Sonic hedgehog, Indian hedgehog and Desert hedgehog), of which Sonic hedgehog (SHH) is the best studied. Activation of $\mathrm{HH}$ signaling is initiated by binding of $\mathrm{HH}$ to the transmembrane receptor Patched (Ptch), resulting in the release of Ptch-mediated suppression of Smoothened (Smo), a G-protein-coupled receptor. Released Smo subsequently activates the Gli family of transcription factors that regulate the expression of $\mathrm{HH}$ target genes (9,17-19), including vascular endothelial growth factor-A (VEGF-A). VEGF-A is considered as one of the most potent angiogenic factors overexpressed in many human cancers (20-24). VEGF-A secreted from tumor cells primarily binds to specific receptors such as VEGFR-2 that are located on vascular endothelial cells (EC) (17,25-27). Binding of VEGF-A to VEGFR-2 in turn triggers a tyrosine kinase signaling cascade that induces EC proliferation, migration, survival, sprouting and tube formation (17,25,28-30). Thus, inhibition of angiogenesis via modulation of SHH signaling could be a major focus for anticancer drug development. 
Hedyotis diffusa Willd (HDW), belonging to the Rubiaceae family, is a medicinal herb widely distributed in Northeast Asia. As a well known traditional Chinese folk-medicine, HDW has long been used as a major component in many traditional Chinese medicine (TCM) formulas for the clinical treatment of various kinds of cancer including CRC (31-33). Recently, we reported that HDW can inhibit cancer growth in vivo and in vitro via suppression of STAT3 pathway $(3,34)$. In addition, we proposed that HDW displays anti-angiogenic activity in vitro (25). However, the precise mechanism of its anticancer activity remains largely unclear. To further elucidate the mechanism of the tumoricidal activity of Hedyotis diffusa Willd, here we evaluated its in vivo effect on colorectal cancer growth and tumor angiogenesis and investigated the underlying molecular mechanisms.

\section{Materials and methods}

Materials and reagents. Dulbecco's modified Eagle's medium (DMEM), fetal bovine serum (FBS), penicillin/streptomycin, Trypsin-EDTA and TRIzol Reagent were purchased from Invitrogen (Carlsbad, CA, USA). SuperScript II reverse transcriptase was obtained from Promega (Madison, WI, USA). CD31, SHH, PTCH-1, SMO, Gli-1, VEGF-A and VEGFR2 antibodies, secondary antibodies were obtained from Santa Cruz Biotechnology (Santa Cruz, CA, USA). All other chemicals, unless otherwise stated, were obtained from Sigma Chemicals (St. Louis, MO, USA).

Preparation of ethanol extract from Hedyotis diffusa Willd $(E E H D W)$. Authentic plant material was purchased from Guo Yi Tang Chinese Herbal medicine store, Fujian, China. The original herb was identified as Hedyotis diffusa Willd (HDW) by Dr Wei Xu at Department of Pharmacology, Fujian University of Traditional Chinese Medicine, China. Ethanol extract of HDW (EEHDW) was prepared as previously described (3). Briefly, $500 \mathrm{~g}$ of HDW was extracted with $5,000 \mathrm{ml}$ of $85 \%$ ethanol using a refluxing method and filtered. The ethanol solvent was then evaporated on a rotary evaporator (Yarong, Model RE-2000, Shanghai, China). The resultant solution was concentrated to a relative density of 1.05 and the dried powder of EEHDW was obtained by a spraying desiccation method using a spray dryer (Buchi, Model B-290, Flawil, Switzerland). The working concentrations of EEHDW were made by dissolving the extract in saline to a concentration of $0.6 \mathrm{~g} / \mathrm{ml}$.

Cell culture and animals. Human colon carcinoma cell line HT-29 was obtained from the American Type Culture Collection (ATCC, Manassas, VA, USA) and grown in DMEM medium supplemented with $10 \%$ serum (Gibco-BRL), $100 \mathrm{U} / \mathrm{ml}$ penicillin and $100 \mu \mathrm{g} / \mathrm{ml}$ streptomycin in a humidified environment with $5 \% \mathrm{CO}_{2}$ at $37^{\circ} \mathrm{C}$. Male $\mathrm{BALB} / \mathrm{C}$ athymic nude mice (with an initial body weight of 20-22 g) were purchased from Shanghai Si-Lai-Ke Experimental Animal Ltd (Shanghai, China). The mice were housed five per cage in specific pathogen-free room in an environment with controlled temperature $\left(22^{\circ} \mathrm{C}\right)$, humidity and a $12 \mathrm{~h}$ light/dark cycle with free access to water and standard laboratory food. All animal treatments were strictly in accordance with international ethical guidelines and the National Institutes of Health Guide concerning the Care and Use of Laboratory Animals, and the experiments were approved by the Institutional Animal Care and Use committee of Fujian University of Traditional Chinese Medicine.

In vivo nude mouse xenograft study. HT-29 cells $\left(5 \times 10^{6}\right)$ mixed with Matrigel (1:1) were subcutaneously injected in the right flank area of athymic nude mice to initiate tumor growth. After 5 days of xenograft implantation, mice were randomly divided into two groups $(n=10)$ and given intra-gastric administration with $3 \mathrm{~g} / \mathrm{kg}$ of EEHDW or saline daily, 6 days a week for 16 days. Tumor diameters were measured at regular intervals with digital calipers and the tumor volume $(\mathrm{V})$ was calculated using the formula: $\mathrm{V}=(\text { width })^{2} \mathrm{x}$ length $\mathrm{x} \pi / 6$. At the end of experiment, the animals were anaesthetized and the tumor issue was removed. Blood was obtained aseptically from orbit. Bloodcontaining tubes were allowed to stand at room temperature for $5 \mathrm{~h}$ and serums were obtained by centrifugation at $3,000 \mathrm{xg}$ for $20 \mathrm{~min}$.

RNA extraction and RT-PCR analysis. Total RNA was isolated from fresh tumor tissues with TRIzol Reagent. Oligo(dT)-primed RNA (1 $\mu \mathrm{g})$ was reverse-transcribed with SuperScript II reverse transcriptase (Promega) according to the manufacturer's instructions. The obtained cDNA was used to determine the mRNA amount of SHH, PTCH, SMO, Gli-1, VEGF-A and VEGFR2 by PCR with Taq DNA polymerase (Fermentas). GAPDH was used as an internal control. Samples were analyzed by gel electrophoresis (1.5\% agarose). The DNA bands were examined using a Gel Documentation System (Bio-Rad, Model Gel Doc 2000, Hercules, CA, USA).

Immunohistochemical staining. After fixing with $10 \%$ formaldehyde for $12 \mathrm{~h}$, tumor samples were processed conventionally for $5-\mu \mathrm{m}$-thick paraffin-embedded tumor slides. The slides were subjected to antigen retrieval and the endogenous peroxidase activity was quenched with hydrogen peroxide. After blocking non-specific proteins with normal serum in PBS, slides were incubated with rabbit polyclonal antibodies against CD31, SHH, PTCH-1, SMO, Gli-1, VEGF-A and VEGFR2 (all in 1:100 dilution, Santa Cruz Biotechnology). After washing with PBS, slides were incubated with biotinylated secondary antibody followed by conjugated HRP-labelled streptavidin (Dako) and then washed with PBS. The slides were then incubated with diaminobenzidine (DAB, Sigma Chemicals) as the chromogen, followed by counterstaining with diluted Harris hematoxylin (Sigma Chemicals). After staining, five high-power fields (x400) were randomly selected in each slide, and the average proportion of positive cells in each field were counted using the true color multi-functional cell image analysis management system (Image-Pro Plus, Media Cybernetics, Bethesda, MD, USA). To rule out any non-specific staining, PBS was used to replace the primary antibody as a negative control.

Statistical analysis. All data are the means of three determinations and data were analyzed using the SPSS package for Windows (Version 11.5). Statistical analysis of the data was 


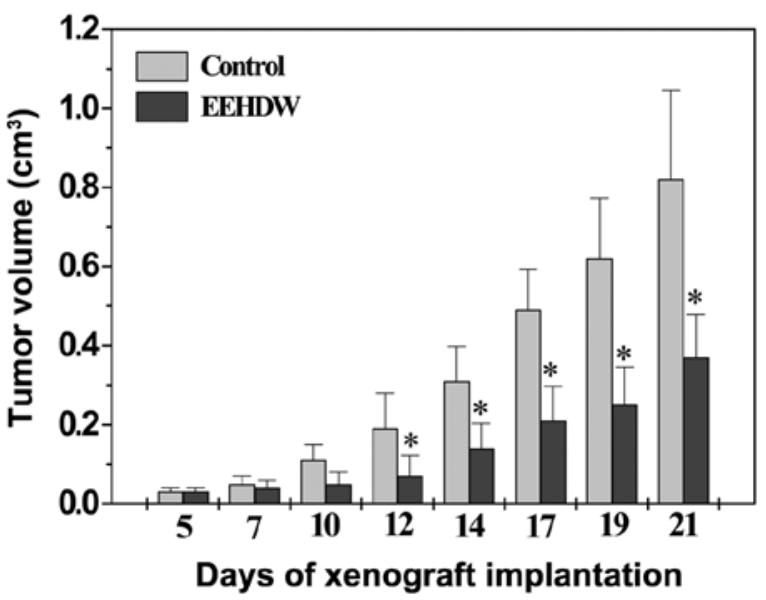

Figure 1. Effect of EEHDW on tumor growth in CRC xenograft mice. After tumor development, the mice were given intra-gastric administration with $3 \mathrm{~g} / \mathrm{kg}$ of EEHDW or saline daily, 6 days a week for 16 days. Tumor volume was measured during the experiment. Data shown are averages with SD (error bars) from 10 mice in each group. ${ }^{*} \mathrm{P}<0.01$ vs. controls.

performed with Student's t-test and ANOVA. Differences with $\mathrm{P}<0.05$ were considered statistically significant.

\section{Results}

EEHDW inhibits tumor growth in colorectal cancer $(C R C)$ xenograft mice. The inhibitory effect of EEHDW on cancer growth was evaluated by measuring tumor volume. We found that administration with EEHDW significantly inhibited tumor growth throughout the study, as compared with the control group (Fig. 1). At the end of experiments, tumor volume per mouse in EEHDW treatment group was $0.37 \pm 0.11 \mathrm{~cm}^{3}$, whereas that in control group was $0.82 \pm 0.23 \mathrm{~cm}^{3}$ (Fig. 1, $\mathrm{P}<0.01)$, demonstrating the therapeutic efficacy of EEHDW against CRC in vivo.

EEHDW displays anti-angiogenic activity in CRC xenograft mice. We performed immunohistochemical staining (IHS) for endothelial cell-specific marker CD31, to determine the effect of EEHDW on intratumoral microvessel density (MVD) that is considered as an indicator of tumor angiogenesis and is associated with cancer growth and progression (17). As shown in Fig. 2, the percentage of CD31-positive cells in tumors from EEHDW-treated or control mice was $24.67 \pm 5.43$ or $35.33 \pm 2.88 \%$, respectively $(\mathrm{P}<0.01)$, suggesting that inhibition of tumor angiogenesis by EEHDW could have contributed to the inhibition of colorectal tumor growth.

EEHDW suppresses SHH pathway in CRC xenograft mice. To determine the effect of EEHDW on SHH pathway, we evaluated mRNA and protein expression levels of the key mediators of this pathway in CRC xenograft tumors using RT-PCR and IHS assay. Data from RT-PCR showed that EEHDW profoundly reduced the mRNA expression of $\mathrm{SHH}$, Ptch-1, Smo and Gli-1 in CRC tumor tissues (Fig. 3A, P<0.01). In a consistent manner, the protein expression of these factors was significantly downregulated by EEHDW treatment. The percentage of cells in the CRC xenograft tumors expressing SHH, Ptch-1, Smo or Gli-1 in the EEHDW-treated mice was $25.67 \pm 7.81,18.00 \pm 3.96,26.00 \pm 4.98$ or $25.17 \pm 5.12 \%$, whereas that in control group was $34.83 \pm 5.31,35.33 \pm 5.24,37.33 \pm 5.99$ or $35.83 \pm 7.30 \%$, respectively (Fig. $3 \mathrm{~B}, \mathrm{P}<0.01$ ). Collectively, these data suggest that EEHDW inhibits the activation of $\mathrm{SHH}$ signaling in colorectal cancer.

EEHDW decreases VEGF and VEGFR2 expression in tumors. To further investigate the mechanism of anti-angiogenic activity of EEHDW, we examined the expression of VEGF-A, a critical target gene of SHH pathway, as well as the expression of its specific receptor VEGFR2. As shown in Fig. 4, EEHDW treatment obviously decreased the mRNA and protein expression levels of VEGF-A and VEGFR2 in CRC tumor tissues $(\mathrm{P}<0.01)$.

\section{Discussion}

Angiogenesis has become an attractive target for anticancer chemotherapy due to its critical role for the progression and metastasis of solid tumors. A number of anti-angiogenic agents are currently in preclinical development, with some of them

\section{Control}

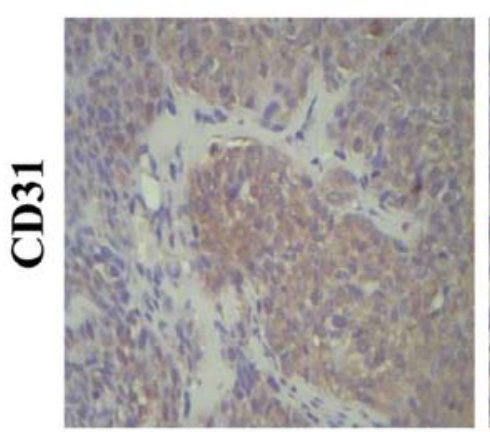

EEHDW

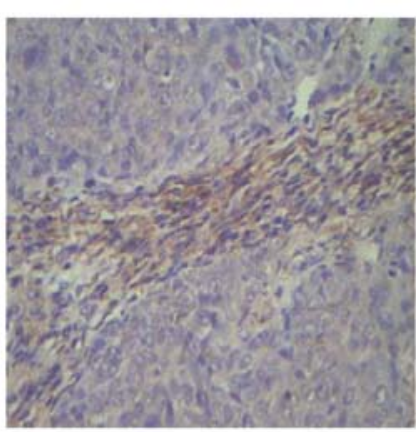

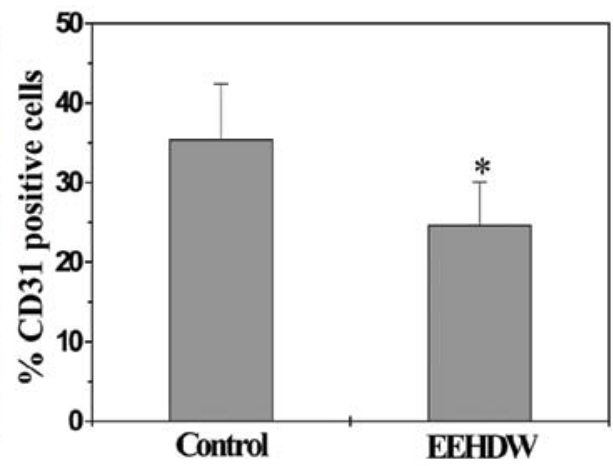

Figure 2. Effect of EEHDW on the intratumoral microvessel density in CRC xenograft mice. Tumor tissues were processed for IHC staining (IHS) for CD31. The photographs are representative images taken at a magnification, x400. Quantification of IHS is represented as percentage of positively-stained cells. Data shown are averages with SD (error bars) from 10 mice in each group. ${ }^{*} \mathrm{P}<0.01$ vs. controls. 

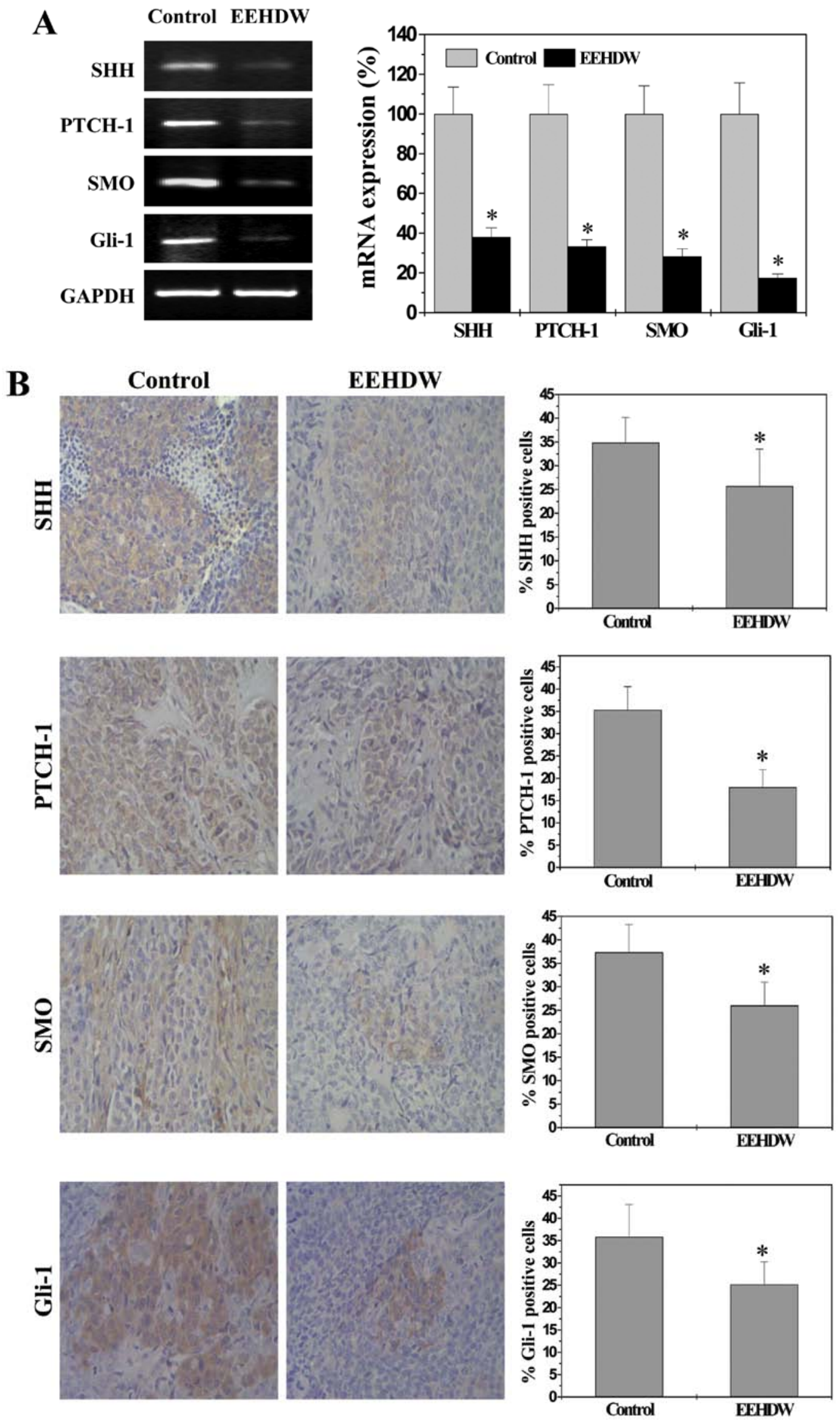

Figure 3. Effect of EEHDW on the activation of SHH pathway in CRC xenograft mice. (A) The mRNA expression levels of SHH, PTCH-1, SMO and Gli-1 were determined by RT-PCR. GAPDH was used as the internal control. The data of densitometric analysis were normalized to the mean mRNA expression of untreated control (100\%). (B) Tumor tissues were processed for IHS for SHH, PTCH-1, SMO and Gli-1. The photographs are representative images taken at a magnification, x400. Quantification of IHS is represented as percentage of positively-stained cells. Data shown are averages with SD (error bars) from 10 mice in each group. ${ }^{*} \mathrm{P}<0.01$ vs. controls. 
A
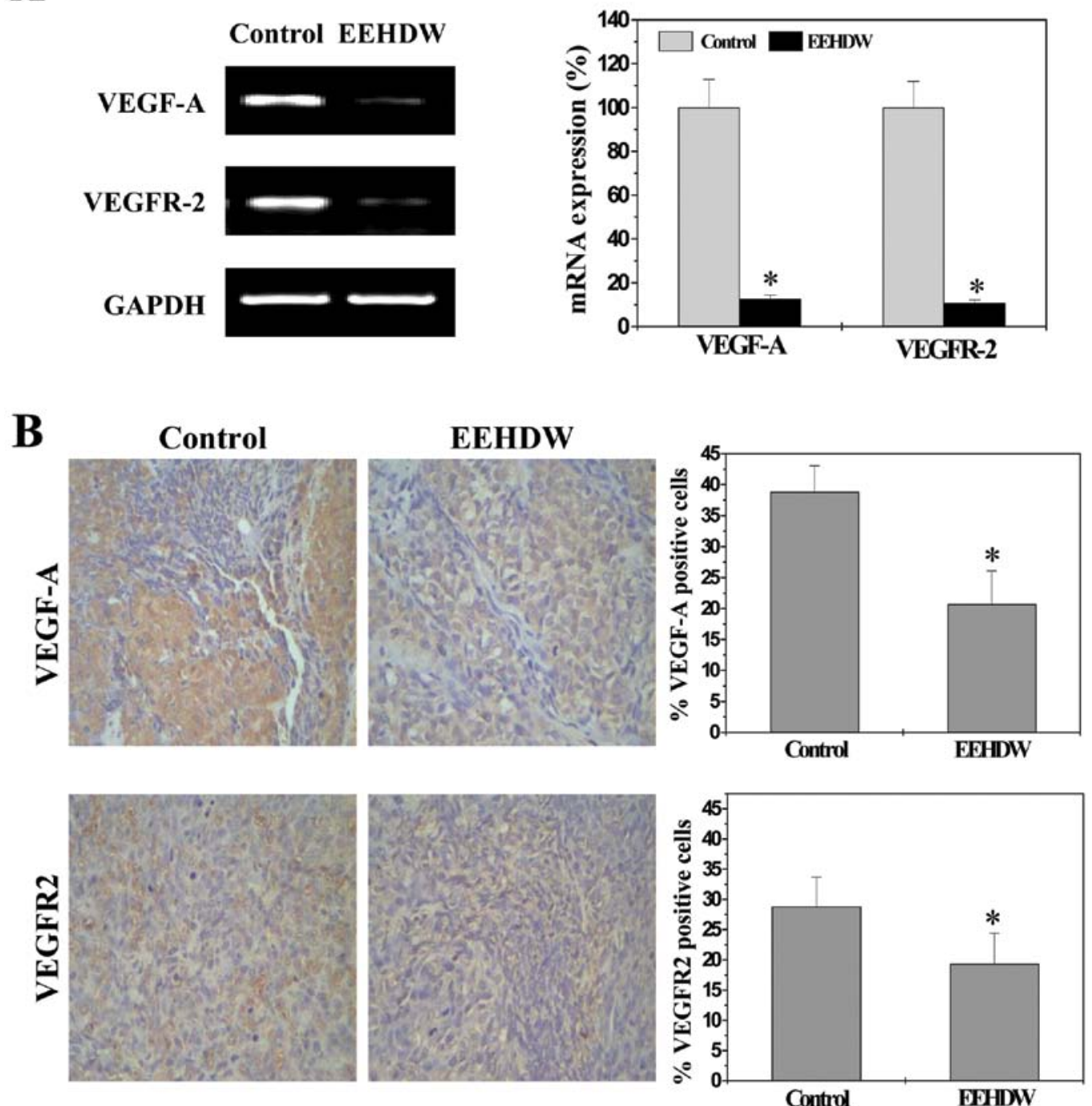

Figure 4. Effect of EEHDW on the expression of VEGF-A and VEGFR2 in CRC xenograft mice. (A) The mRNA expression levels of VEGF-A and VEGFR2 were determined by RT-PCR. GAPDH was used as the internal control. The data of densitometric analysis were normalized to the mean mRNA expression of untreated control (100\%). (B) Tumor tissues were processed for IHS for VEGF-A and VEGFR2. The photographs are representative images taken at a magnification, $\mathrm{x} 400$. Quantification of IHS is represented as percentage of positively-stained cells. Data shown are averages with SD (error bars) for 10 mice in each group. "P $<0.01$ vs. controls.

now entering the clinic trials. However, due to the intrinsic cytotoxicity against non-tumor associated endothelial cells, long-term use of angiogenesis inhibitors usually causes cardiovascular complications such as impaired wound healing, bleeding, hypertension and thrombosis (35-37). Therefore, discovering novel antitumor agents with minimal adverse effects is needed. Natural products, including traditional Chinese medicine (TCM), have relatively fewer side-effects and have been used clinically to treat various kinds of diseases including cancer for thousands of years. Hedyotis diffusa Willd (HDW), a well known traditional Chinese folk-medicine, has long been used as a major component in several TCM formulas for the clinical treatment of various kinds of cancer including colorectal cancer (CRC) (3). Recently, we reported that HDW can inhibit cancer growth in vivo and in vitro via suppression of STAT3 pathway $(3,34)$ and displays anti-angiogenic activity in vitro (25). However, the mode of action for its antitumor effect is still largely unknown.

In the present study we confirmed that the ethanol extract of Hedyotis diffusa Willd (EEHDW) could inhibit cancer growth in vivo, consistent with our previous study (34). More importantly, by using immunohistochemical (IHC) staining for the endothelial cell-specific marker CD31 we found that EEHDW significantly reduced the intratumoral microvessel density (MVD), demonstrating its in vivo anti-angiogenic activity. Tumor angiogenesis is tightly regulated by Sonic hedgehog (SHH) pathway. Activation of $\mathrm{HH}$ signaling upregulates the expression of VEGF-A which is the strongest angiogenesis stimulator $(20,21,29,30,38)$. VEGF-A exerts its pro-angiogenic function via binding to its specific receptors including VEGFR2 which is located on vascular endothelial cells (EC) (25-29), leading to series of angiogenic processes $(29,30)$. In this study we found that EEHDW treatment inhibited the activation of 
SHH pathway in tumor tissues since EEHDW significantly suppressed the mRNA and protein expression of several key mediators of SHH signaling, including SHH, PTCH-1, SMO and GLi-1. Consistently, administration of EEHDW profoundly reduced the expression of VEGF-A and VEGFR2 in vivo, at both transcriptional and translational levels.

In conclusion, here for the first time we demonstrate that Hedyotis diffusa Willd inhibits colorectal cancer growth in vivo via inhibition of SHH-mediated tumor angiogenesis, which may in part explain its anticancer activity.

\section{Acknowledgements}

This study was sponsored by the Natural Science Foundation of Fujian Province of China (2010J01195), the Research Foundation of Education Bureau of Fujian Province of China (JA10162) and the National Natural Science Foundation of China (81073097).

\section{References}

1. Jemal A, Bray F, Center MM, Ferlay J, Ward E and Forman D: Global cancer statistics. CA Cancer J Clin 61: 69-90, 2011.

2. Gustin DM and Brenner DE: Chemoprevention of colon cancer: current status and future prospects. Cancer Metast Rev 21: 323-348, 2002.

3. Lin J, Chen Y, Wei L, Chen X, Xu W, Hong Z, Sferra TJ and Peng J: Hedyotis diffusa Willd extract induces apoptosis via activation of the mitochondrion-dependent pathway in human colon carcinoma cells. Int J Oncol 37: 1331-1338, 2010.

4. Gordaliza M: Natural products as leads to anticancer drugs. Clin Transl Oncol 9: 767-776, 2007.

5. Ji HF, Li XJ and Zhang HY: Natural products and drug discovery. Can thousands of years of ancient medical knowledge lead us to new and powerful drug combinations in the fight against cancer and dementia? EMBO Rep 10: 194-200, 2009.

6. Folkman J: Tumor angiogenesis: therapeutic implications. N Engl J Med 285: 1182-1186, 1971.

7. Folkman J: Angiogenesis. Annu Rev Med 57: 1-18, 2006.

8. Cook KM and Figg WD: Angiogenesis inhibitors: current strategies and future prospects. CA Cancer J Clin 60: 222-243, 2010.

9. Ingham PW, Nakano Y and Seger C: Mechanisms and functions of Hedgehog signalling across the metazoa. Nat Rev Genet 12 : 393-406, 2011

10. Zhang S, Wang Y, Mao JH, Hsieh D, Kim IJ, Hu LM, Xu Z, Long H, Jablons DM and You L: Inhibition of CK $2 \alpha$ downregulates Hedgehog/Gli signaling leading to a reduction of a stem-like side population in human lung cancer cells. PLoS One 7: e38996, 2012.

11. Theunissen JW and de Sauvage FJ: Paracrine Hedgehog signaling in cancer. Cancer Res 69: 6007-6010, 2009.

12. Mazumdar T, DeVecchio J, Shi T, Jones J, Agyeman A and Houghton JA: Hedgehog signaling drives cellular survival in human colon carcinoma cells. Cancer Res 71: 1092-1102, 2011.

13. Yoshikawa K, Shimada M, Miyamoto H, Higashijima J, Miyatani T, Nishioka M, Kurita N, Iwata T and Uehara H: Sonic hedgehog relates to colorectal carcinogenesis. J Gastroenterol 44: 1113-1117, 2009.

14. Varnat F, Duquet A, Malerba M, Zbinden M, Mas C, Gervaz P and Ruiz i Altaba A: Human colon cancer epithelial cells harbour active HEDGEHOG-GLI signalling that is essential for tumour growth, recurrence, metastasis and stem cell survival and expansion. EMBO Mol Med 1: 338-351, 2009.

15. Das S, Tucker JA, Khullar S, Samant RS and Shevde LA: Hedgehog signaling in tumor cells facilitates osteoblastenhanced osteolytic metastases. PLoS One 7: e34374, 2012.

16. Sahebjam S, Siu LL and Razak AA: The utility of Hedgehog signaling pathway inhibition for cancer. Oncologist 17 : 1090-1099, 2012.
17. Wei L, Lin J, Xu W, Cai Q, Shen A, Hong Z and Peng J: Scutellaria barbata D. Don inhibits tumor angiogenesis via suppression of Hedgehog pathway in a mouse model of colorectal cancer. Int J Mol Sci 13: 9419-9430, 2012.

18. Lum L and Beachy PA: The Hedgehog response network: sensors, switches, and routers. Science 304: 1755-1759, 2004.

19. Varjosalo $M$ and Taipale J: Hedgehog: functions and mechanisms. Genes Dev 22: 2454-2472, 2008.

20. Pola R, Ling LE, Silver M, Corbley MJ, Kearney M, Pepinsky RB, Shapiro R, Taylor FR, Baker DP and Asahara T: The morphogen Sonic hedgehog is an indirect angiogenic agent upregulating two families of angiogenic growth factors. Nat Med 7: 706-711, 2001.

21. Claret S, Sanial M and Plessis A: Evidence for a novel feedback loop in the Hedgehog pathway involving smoothened and fused. Curr Biol 17: 1326-1333, 2007.

22. Kerbel RS: Tumor angiogenesis. N Engl J Med 358: 2039-2049, 2008.

23. Prasad S, Yadav VR, Sung B, Reuter S, Kannappan R, Deorukhkar A, Diagaradjane P, Wei C, Baladandayuthapani V, Krishnan S, Guha S and Aggarwal BB: Ursolic acid inhibits growth and metastasis of human colorectal cancer in an orthotopic nude mouse model by targeting multiple cell signaling pathways: chemosensitization with capecitabine. Clin Cancer Res 18: 4942-4953, 2012.

24. Dai J, Wan S, Zhou F, Myers RE, Guo X, Li B, Fu X, Palazzo JP, Dou K, Yang $\mathrm{H}$ and Xing J: Genetic polymorphism in a VEGF-independent angiogenesis gene ANGPT1 and overall survival of colorectal cancer patients after surgical resection. PLoS One 7: e34758, 2012.

25. Lin J, Wei L, Xu W, Hong Z, Liu X and Peng J: Effect of Hedyotis diffusa Willd extract on tumor angiogenesis. Mol Med Rep 4: 1283-1288, 2011.

26. Ferrara N, Gerber HP and LeCouter J: The biology of VEGF and its receptors. Nat Med 9: 669-676, 2003.

27. Shigami SI, Arii S, Furutani M, Niwano M, Harada T, Mizumoto M, Mori A, Onodera $\mathrm{H}$ and Imamura M: Predictive value of vascular endothelial growth factor (VEGF) in metastasis and prognosis of human colorectal cancer. Br J Cancer 78: 1379-1384, 1998.

28. Gille H, Kowalski J, Li B, LeCouter J, Moffat B, Zioncheck TF, Pelletier N and Ferrara N: Analysis of biological effects and signaling properties of Flt-1 (VEGFR-1) and KDR (VEGFR-2). A reassessment using novel receptor-specific vascular endothelial growth factor mutants. J Biol Chem 276: 3222-3230, 2001.

29. Ferrara N: Role of vascular endothelial growth factor in physiologic and pathologic angiogenesis: therapeutic implications. Semin Oncol 29: 10-14, 2002.

30. Risau W: Mechanisms of angiogenesis. Nature 386: 671-674, 1997.

31. Ren F, Liu G, Zhang L and Niu G: Studies on chemical constituents of Hedyotis diffusa Willd. Chin Pharm J 40: 502-504, 2005.

32. Ahmad R, Shaari K, Lajis NH, Hamzah AS, Ismail NH and Kitajima M: Anthraquinones from Hedyotis capitellata. Phytochemistry 66: 1141-1147, 2005.

33. Li C, Xue X, Zhou D, Zhang F, Xu Q, Ren L and Liang X: Analysis of iridoid glucosides in Hedyotis diffusa by highperformance liquid chromatography/electrospray ionization tandem mass spectrometry. J Pharm Biomed Anal 48: 205-211, 2008.

34. Cai Q, Lin J, Wei L, Zhang L, Wang L, Zhan Y, Zeng J, Xu W, Shen A, Hong Z and Peng J: Hedyotis diffusa Willd inhibits colorectal cancer growth in vivo via inhibition of STAT3 signaling pathway. Int J Mol Sci 13: 6117-6128, 2012.

35. Chen HX and Cleck JN: Adverse effects of anticancer agents that target the VEGF pathway. Nat Rev Clin Oncol 6: 465-477, 2009.

36. Zangari M, Fink LM, Elice F, Zhan F, Adcock DM and Tricot GJ: Thrombotic events in patients with cancer receiving antiangiogenesis agents. J Clin Oncol 27: 4865-4873, 2009.

37. Higa GM and Abraham J: Biological mechanisms of bevacizumab-associated adverse events. Expert Rev Anticancer Ther 9: 999-1007, 2009

38. Jain RK: Tumor angiogenesis and accessibility: role of vascular endothelial growth factor. Semin Oncol 29: 3-9, 2002. 